\title{
An Artificial Neural Network Model as a Tool to Identify the Anaerobic Threshold during Dynamic Physical Exercise
}

\author{
AC Silva Filho ${ }^{1}, \mathrm{RM} \mathrm{Souza}^{2}, \mathrm{~L}$ Gallo $\mathrm{Jr}^{3}$, LO Murta $\mathrm{Jr}^{3}$ \\ ${ }^{1}$ Centro Universitário de Franca, Franca, Brasil \\ ${ }^{2}$ Universidade de Franca, Franca, Brasil \\ ${ }^{3}$ Universidade de São Paulo, Ribeirão Preto, Brasil
}

\begin{abstract}
Anaerobic threshold is one of the most important parameters used in exercise physiology. It signals a power value during dynamic physical exercise where anaerobic energy formation for muscle contraction is added to the aerobic counterpart-what allows the quantification of aerobic capacity. In this study, we describe the development and validation of an artificial neural network model to identify anaerobic threshold based on electrocardiogram $R-R$ interval time series collected during physical exercise tests applied in healthy subjects. The results showed that the artificial neural network had its best performance in gradual increasing power. Scatter plot and ROC curve was constructed showing high correlation $(r=0.93)$, and good accuracy (area under the ROC curve $=0.9851$ ) when compared to autoregressive integrated moving average (ARIMA) statistical method.
\end{abstract}

\section{Introduction}

This paper presents a new non-invasive method to identify a very important physiologic parameter, the anaerobic threshold, which allows quantifying oxygen transport during an exercise, and, therefore, the exercise evolution with respect to duration and intensity [1]. R-R intervals time series were acquired from electrocardiograms and, then, the series were analyzed using an artificial neural network for such task through personal computers. Artificial neural networks have been used as a decision support system [2] in the clinical environments. The heart rate variability has been used as a parameter for non-invasive system development aimed to solve problems of pattern recognition in medicine [3].

The physical exercise, as a spontaneous activity of daily life, involves complex physiological processes, which nowadays are not completely known. Most exercises result on movements of body segments and living organisms, related to different degrees of force generation to guarantee the survival of such organism in the environments. The muscle contraction involves the transformation of chemical energy stored as a molecule, adenosine tri-phosphate (ATP), into mechanical energy plus heat. In order to maintain this energy transformation for several seconds, or to be repeated for short intervals, the whole physiological system must be activated [4].

There are several kinds of muscle contraction, and therefore, different kinds of exercises. It is important to have in mind the differences between isometric and isotonic effort. The first one, also called static effort, does not result in changes to muscle length, what occurs in situations like weight lifting. The second one, also called dynamic effort, is followed by movement of muscular segment, and thus, by skeletal displacement. The nervous, cardiovascular, respiratory and muscular systems can be considered subsystems that transport the oxygen from the air into the muscular mitochondria and, on the other hand, the carbon dioxide, produced by aerobic metabolism, out of muscular cells. Carbon dioxide is the final result of the aerobic metabolism that constitutes in the most efficient production of energy process.

The magnitude of the cardiorespiratory variables induced by the exercise depends on many factors. When a person makes exercise in a cycloergometer, with the power increasing gradually, the consumption of oxygen (VO2) raises in linear way, until the point where additions of power levels do not modify the VO2; in these conditions, the $\mathrm{VO} 2$ reached its maximum value. The VO2 maximum is one of the most reliable parameters to measure the maximum magnitude of the oxygen transport involved in the chain of the physiological processes previously mentioned. In healthy individuals and patients with cardiorespiratoy diseases this point is rarely reached, because people interrupt the 
exercise before this limit, due to the discomfort manifested as fatigue or muscular pain. In these circumstances, it is possible to get only VO2 peak, which always corresponding to a lower value compared to $\mathrm{VO} 2$ maximum.

Due to these facts, the VO2 peak, generally, depends much more on the perception intensity (fatigue, muscles pain and air lack) of the effort magnitude, at a defined intensity of applied power, than on the saturation of the physiological systems. Therefore, it cannot consist in an objective measure of the transport of $\mathrm{O} 2$ in the dynamic exercise. The Anaerobic Threshold (AT), due to the combination of some metabolic and neuro-humoral processes, still not totally known, is the starting point of the rise of the blood latic acid, which is related to the unbalance between its muscular production and its metabolization processes by the liver and other tissues. There is a high level correlation between the maximum VO2 and the AT, which allows estimating maximum VO2 from the AT. From metabolic and neuro-humoral point of view, it is worth to state that the AT has a great physiological importance, being the limit between two different functional states. The study of the transport of $\mathrm{O} 2$ in healthy individuals and patients with cardiorespiratory diseases had great advances in the last two decades, thanks to the possibility to measure the AT from respiratory methods, using automatic and noninvasive procedures.

Some non-invasive methods and protocols have been used with the purpose of measuring the AT. The most commonly used methods nowadays that satisfy the required sensitivity and accuracy are based on the change in the pattern of respiratory and metabolic responses in AT. In such studies, it is mandatory to use equipment that allows the measurement of the mentioned variables at each breath, or the average value for each 15 seconds. This equipment is expensive, which limits its use to few centers. In exercise studies, using discontinuous step test, it has been shown that below AT a reduction of parasympathetic (vagal) efferent occurred in the sinusal node, what causes a fast rise in heart rate, with a full stabilization of it around one minute of the effort. Besides the vagal system participation, at powers above the AT, stimulation of sympathetic efferent occurred proportional to the intensity of the power applied, causing a slow rise in the in HR, starting at the first effort minute until the end of the effort; in these conditions there is no stabilization of this variable values during the physical effort.

In this work, we use the artificial neural network technique, which is an adaptive non-specialist system, already used by other authors for clinical foreseeing for a particular disease $[5,6]$ or for various diseases. In this context, this work was developed aimed to identify the anaerobic threshold in healthy subjects.

The experimental data were collected with two protocols: the subjects were submitted to a physical effort by a cycloergometer, where the load varied in alternate way in first protocol, and progressively in a second one. The present work has the aim to develop an artificial neural network model to classify R-R time series according to anaerobic threshold from healthy subjects in dynamic effort test, verifying the feasibility of such system as a decision support. The system validation is accomplished with the studied data set collected in alternating effort protocol that showed a better generalization performance by the neural network in finding the anaerobic threshold.

\section{Methods}

It has been chosen fourteen input variables to the proposed neural network. The first three parameters calculated in all the series were first order statistical estimations: the RR time series mean value, the standard deviation and the root mean square of the side difference (RMSSD). All written in equations 1, 2 and 3, respectively:

$$
\begin{gathered}
\bar{x}=\frac{\sum_{i=1}^{N}\left(x_{i}\right)}{N} \\
\sigma=\sqrt{\frac{\sum_{i=1}^{N}\left(R R_{i}-\overline{R R}\right)^{2}}{N}} \\
R M S S D=\sqrt{\frac{\sum_{i=1}^{N-1}\left(R R_{i}-R R_{i+1}\right)^{2}}{N-1}}
\end{gathered}
$$

In addition to these three calculated parameters, it was also submitted to the net the respective power of the bicycle in each RR time series. The ten order autoregressive model for each series was calculated and added to these parameters as input of the net, totalizing fourteen input parameters. to the neural net. For training and model output testing, the neural net output was compared to the results obtained using ARIMA method for the same data set.

The pattern classification in this study was done with neural nets with one and two intermediate layers, one input layer, and one output layer. The first layer was constituted of fourteen input parameters. Architectures 
with an intermediate layer of $13,15,17,19,21,23,25$, 27, 29 and 31 neurons were implemented and analyzed, as well as a net with two intermediate layers with 17 and 19 neurons in each one of them. It was chosen, in the present study, a hyperbolic tangent as transfer function for all the neurons of artificial neural network.

The original data set included more than 300 series: 147 of gradual increasing powers and 153 of alternating powers. For each studied protocol, the series were divided in three subgroups: training, validation and test - where $80 \%$ was reserved for training phase, $10 \%$ for the validation phase, and $10 \%$ for the test phase. The data from these subgroups were chosen randomly to improve the performance. Two algorithms were used for training the nets: 1) Rprop, backpropagation with adaptative learning rate; and 2) backpropagation with momentum. The parameters for every algorithm were: $1.0 \mathrm{e}^{-10}$ for minimum performance gradient; 0.01 for learning rate and a momentum rate of 0.9 . The training was repeated five times, for each selected network architecture, with random initial weights, generating five distinct parts of the training, validation and test sets to attenuate the weights initializing effect, which may results on net stabilization in local minimums and, also provide a more accurate evaluation of the net performance.

\section{Results}

The chosen model using the neural network technique has advantages from other mathematical models due to its simplicity and required computation time in calculations. The matrices of weights and biases of the best net found for each tested set had been stored after the training. The model considered for Neural Nets is shown in figure 1. The best performance neural net architecture was with 14 neurons in input layer, a neuron in the output layer, and an intermediate layer with 29 neurons. All the neurons of a layer were connected to all the neurons of the following layer. The transfer function for all the neurons of the net was set to hyperbolic tangent. The results of performance from three different experiments of the net training with gradual discontinuous powers are shown in Figures 2 and 3. The correlation coefficients of the sets of training, validation and test were $0.896,0.854$ and 0.716 , respectively. The accuracy of the net was measured by the area under the ROC curve plotted for the best performance architecture and is shown in Figure 4. The experimental data was fitted to a exponential curve shown in equation 4 , with the error less than $7.4 \times 10^{-2}$ :

$$
y=0.99-0.38 e^{\left(-\frac{x}{0.04}\right)}
$$

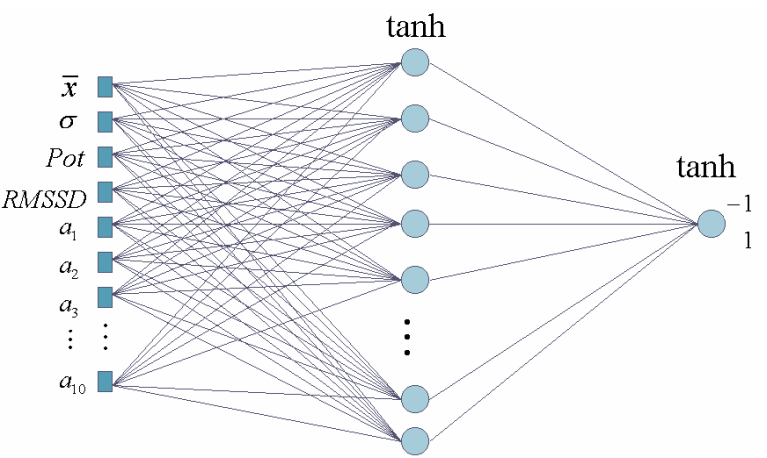

Fig 1: The neural network model

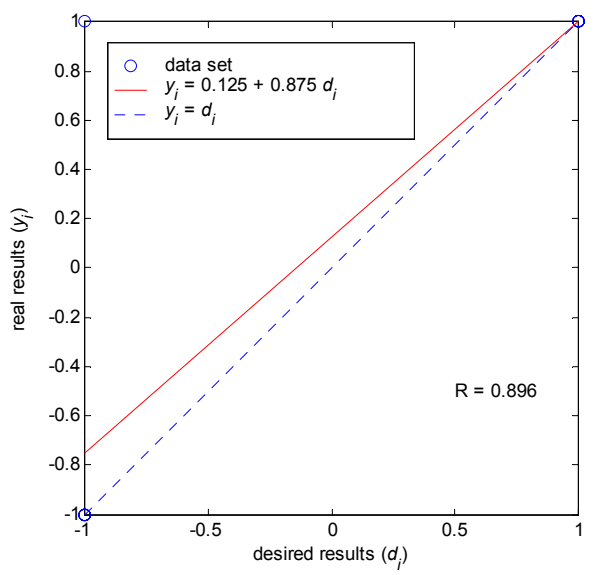

Fig 2: Linear regression of the real results against desired results for the training set of discontinuous increasing power protocol.

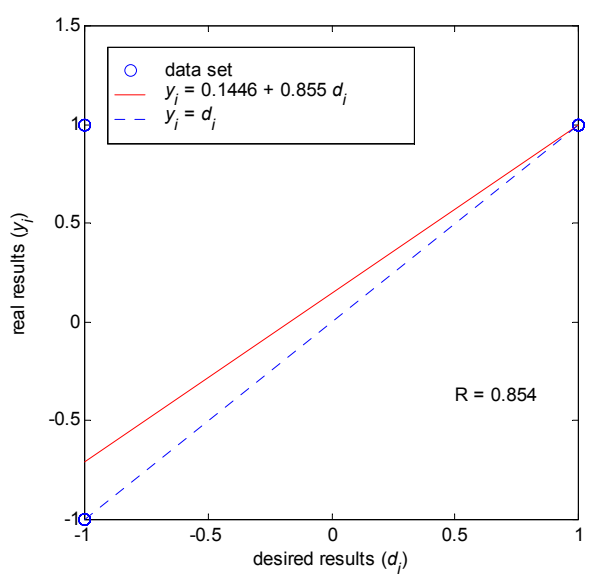

Fig 3: Linear regression of the real results against desired results for the validation set of discontinuous increasing power protocol. 


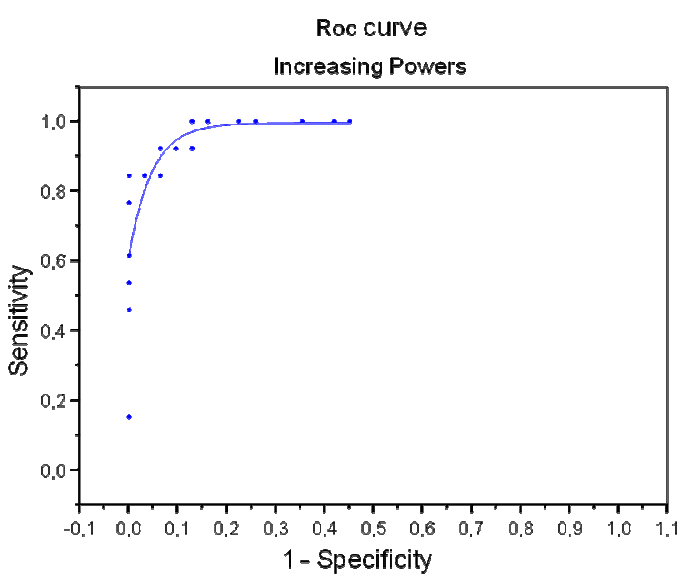

Fig 4: The ROC curve plotted for the best performance architecture.

The area under the ROC curve for alternate power is 0.9851 . The training, validation and test errors for the best architecture found in the training were $14.39 \%, 0 \%$ and $25.60 \%$, respectively. The standard deviation for the sets of training, validation and test were $0.1959,0$ and 0.2560 respectively. These low deviation values most likely indicate that the net has found the global minimum in weights space during training.

\section{Discussion and conclusions}

The anaerobic threshold can be measured directly, with invasive procedures but we have been looking for a non-invasive way of doing the task. A first trial was made using an Auto Regressive Integrated Moving Average Model (ARIMA) [7-9], followed by a second trial that used the Kolmogorov-Sinai entropy (K-S) $[10,12]$ and a third one that used the decorrelation time lag [13].

Our results have shown a reasonable performance of the selected neural network for detecting anaerobic threshold during exercise for data coming obtained from gradual increasing power protocol. Since the area under curve ROC represents $98.51 \%$ of the total area, we can say that the Anaerobic Threshold can be identified using an Artificial Neural Network technique for this protocol, due to the little amount of involved volunteers in the tests the result can be considered a good one (future works should consider more individuals). The high accuracy level, represented by the area under the curve is encouraging. Compared to ARIMA method, which needs statistical specialist monitoring, this method has the advantage of being automated, implying a higher independence of the physician or another health professional. Another advantage for the use of Neural Nets is the reduced time of necessary computation compared to methods of nonlinear analysis.

\section{Acknowledgements}

\author{
Uni-FACEF, FAEPA.
}

\section{References}

[1] Wasserman $\mathrm{K}$ et al. Principles of Exercise Testing and Interpretation, Philadelphia: Lippincott Williams and Wilkins, 1999.

[2] Frize $M$ et al. Clinical decision support systems for intensive care units: using artificial neural networks. Medical Engineering \& Physics 2001; 23:217-225.

[3] Maglaveras $\mathrm{N}$ et al. ECG pattern recognition and classification using non-linear transformations and neural networks: A review. International Journal of Medical Informatics 1998;52:191-208.

[4] Mcardle WD et al. Essentials of exercise physiology. 1994, Lea and Febiger, Philadelphia.

[5] Mobley BA et al. Predictions of coronary artery stenosis by artificial neural network. Artificial Intelligence in Medicine 200;18: 187-203.

[6] Azuaje F et al. Predicting coronary disease risk based on short-term R-R interval measurements: a neural network approach. Artificial Intelligence in Medicine 1999; 15: 275-299.

[7] Gallo Jr L et al. Control of heart rate during exercise in health and disease. Brazilian Journal of Medicine and Biological Research 1995; 28: 1179-84.

[8] Box GEP, Pierce DA. Distribution of residual autocorrelations in autoregressive integrated moving average time-series models. Journal of American Statistical Association 1970; 65: 1509-26.

[9] Box GEP, Jenkins GM. Time Series Analysis: Forecasting and Control. San Francisco: Holden- Day Pub, 1976.

[10] Silva FMHSP et al. Identification of anaerobic threshold during dynamic exercise in healthy man using Kolmogorov-Sinai entropy. Computers in Cardiology 2005; 32:731-734.

[11] Silva FMHSP et al. Is the heart more organized during the exercise? Bollettino Chimico Farmaceutico, 1999; 138:LXXI.

[12] Silva FMHSP et al. Characterization of anaerobic threshold in dynamical physical exercise of healthy men. (in) Accessibility and Quality of Health Services, Berlin, 2004:259-268.

[13] Silva Filho, AC et al. Using the lag of Autocorrelation Function in order to Identify the Anaerobic Threshold During Dynamic Physical Exercise. Computers in Cardiology 2006; 33:625-628.

Address for correspondence

Antônio Carlos da Silva Filho

Uni-FACEF, Centro Universitário de Franca

Av. Dr. Ismael Alonso Y Alonso 2400, Franca - SP.

CEP: 14.403-430, Brazil. E-mail: acdasf@facef.br 